\title{
Dípteros asociados a la floración del aguacate Persea americana Mill cv. Hass en Cauca, Colombia
}

Dipterans associated to the flowering of the avocado Persea americana Mill cv. Hass in Cauca, Colombia

\section{Diana Carabalí-Banguero, James Montoya-Lerma y Arturo Carabalí-Muñoz}

\section{Resumen}

Existe una alta diversidad de insectos asociados a la floración del aguacate. Aunque las abejas son los principales polinizadores del cultivo, especies de distintos gremios tróficos frecuentan sus inflorescencias y poco se conoce sobre su identidad taxonómica. En el municipio de Morales, Cauca, Colombia, se seleccionaron dos parcelas, en las cuales se escogieron aleatoriamente cuatro árboles de aguacate y, cada hora, durante 20 minutos, se realizó la captura de insectos con una jama y el registro de su comportamiento en 10 inflorescencias/árbol entre las 07:00-17:00 horas. Se evaluaron semanalmente los periodos de visita a las flores y la carga polínica de los insectos. Las evaluaciones se efectuaron durante dos épocas de floración. Se encontró alta diversidad de insectos, siendo mayor en los dípteros Lucilia eximia, Chrysomya putoria, Palpada scutellaris y Ornidia obesa. Los insectos presentaron picos de visita floral entre las 10:00-11:00 y 14:00-15:00 horas y, aunque con una baja cantidad de polen en sus cuerpos, se observaron colectando néctar, en cópula o posados en las flores. Esta investigación revela que existe una alta diversidad de dípteros asociados a la floración de Persea americana que puede contribuir a la dispersión del polen en los huertos y diversas funciones biológicas.

Palabras clave. Aguacate. Diptera. Diversidad. Insectos. Polinizadores.

\begin{abstract}
There is a high diversity of insects associated to the flowering of avocado plants. Although bees are the main pollinators of the crop, species of different trophic guilds visit avocado inflorescences, yet little is known about them. In the municipality of Morales, Cauca, Colombia, two plots were selected and four trees were randomly selected from each one. Insects were collected with a net every hour for 20 minutes and the behavior of visiting insects was recorded in 10 inflorescences/tree. Insect collecting and observations were carried between 07:00-17:00 hours. The periods of visit to the flowers and the pollen load of the insects were evaluated weekly. Evaluations were carried out during two flowering periods. A high diversity of species was found associated with avocado inflorescences, being particularly higher in the order Diptera, with species such as Lucilia eximia, Chrysomya putoria, Palpada scutellaris and Ornidia obesa. Insect floral visitors presented peaks between 10:00-11:00 and 14:00-
\end{abstract}


15:00 hours. Although a low amount of pollen on their bodies was observed, insects were collecting nectar, copulating or perched on flowers. This research shows that there is a high diversity of dipterans associated with the flowering of Persea americana that could contribute to pollen dispersal in orchards and associated biological functions.

Keywords. Avocado. Diptera. Diversity. Insects. Pollinators.

\section{Introducción}

El aguacate Persea americana Mill. (Lauraceae) es un frutal perenne originario de Centroamérica (Peña, 2003; Pérez-Balam et al., 2012). Gracias a que presenta protoginia dicogamia sincronizada, un comportamiento floral único, evita la autopolinización. Las flores son pequeñas, de aproximadamente $1 \mathrm{~cm}$, tienen las estructuras reproductivas expuestas y secretan néctar de nectarios o estaminodios (Davenport, 1986; Wysoki et al., 2002; Peña, 2003). La exposición de las estructuras reproductivas y nectaríferas facilita la obtención de recursos nutritivos por los insectos y la visita de una amplia gama de visitantes florales, entre los que se encuentran abejas, avispas y dípteros (Ish-Am et al., 1999; Wysoki et al., 2002; Peña, 2003).

En el centro de origen de $P$. americana, en México, existen cerca de 70 especies de insectos asociados a la floración del cultivo (Ish-Am et al., 1999) pertenecientes a los órdenes: Diptera, Coleoptera, Hemiptera, Hymenoptera, Lepidoptera, Neu roptera y Thysanoptera (Wysoki et al., 2002). Estos presentan una contribución diferencial a la polinización de la especie de acuerdo con su frecuencia de visitas, carga polínica y las regiones del cuerpo que hacen contacto con las estructuras reproductivas de la flor (Ish-Am et al., 1999; Wysoki et al., 2002). Aunque se estima que las especies de Hymenoptera, tanto abejas nativas como la introducida Apis mellifera L. (Apidae), son las polinizadoras más efectivas de este frutal (IshAm et al., 1999; Wysoki et al., 2002), actualmente se conoce muy poco sobre el papel polinizador de otros insectos, en particular dípteros, frecuentes en el cultivo.
Los dípteros sobresalen como insectos importantes en la polinización de especies cultivadas, como la fresa Fragaria sp., cebolla Allium sp., Brassica sp., apio Apium graveolens, pimientos Capsicum annuum en áreas templadas y para algunas especies de plantas con flores que son difíciles de polinizar manualmente (Rader et al., 2013; Inouye et al., 2015). En Suráfrica Rhyncomyia forcipata Villeneuve (Calliphoridae) y Musca domestica L. (Muscidae) se encuentran entre los dípteros más abundantes en flores de aguacate (Eardley y Mansell, 1996). En áreas tropicales, cultivos de cacao, mango y marañón son polinizados por dípteros (Inouye et al., 2015). En México, se reportaron 45 especies de Diptera asociadas a la floración del cultivo, siendo las más importantes dentro de Diptera los Syrphidae: Palpada mexicana Fabricius, Eristalis tenax L. y los Calliphoridae: Chrysomya megacephala Fabricius, Compsomyiops callipes Bigot y Phaenicia mexicana Macquart (Castañeda-Vildózola et al., 1999).

Un amplio número de estudios se ha enfocado en la actividad de las abejas como polinizadores de diferentes especies de plantas silvestres y cultivadas como $P$. americana, sin embargo, la información científica publicada sobre el rol que como visitantes florales y polinizadores tienen otras especies, como los dípteros, es aún escasa. Esta investigación tuvo como propósito identificar dípteros visitantes de la flor de $P$. americana cv. Hass, y reconocer su papel como potenciales polinizadores asociados al cultivo. 


\section{Materiales y métodos}

Área de estudio. En el área de estudio ubicada en el municipio de Morales, Cauca, Colombia, se seleccionaron 2 parcelas experimentales sembradas en monocultivos de $P$. americana cv. Hass, de 6 años de edad, injertados sobre un patrón antillano. Los huertos se denominan: El Recuerdo $\left(02^{\circ} 48^{\prime} 34,5^{\prime \prime} \mathrm{N}, \quad 76^{\circ} 37^{\prime} 15,8^{\prime \prime} \mathrm{O}\right.$, altitud: $1600 \mathrm{~m}$ s.n.m) y San Isidro $\left(02^{\circ} 47^{\prime} 48,26^{\prime \prime} \mathrm{N}, 76^{\circ} 36^{\prime} 34,5^{\prime \prime} \mathrm{O}\right.$, altitud: $1600 \mathrm{~m}$ s.n.m), en adelante referidos como P-1 y P-2, respectivamente. La temperatura promedio es de $21^{\circ} \mathrm{C}( \pm 2,62)$ y humedad relativa de $79 \%( \pm 9,94)$. En las zonas aledañas a los huertos predomina los cultivos de café Coffea arabica L. (Rubiaceae), lulo Solanum quitoense Lam. (Solanaceae) y los predios son contiguos a un bosque lineal ribereño (Figura 1).

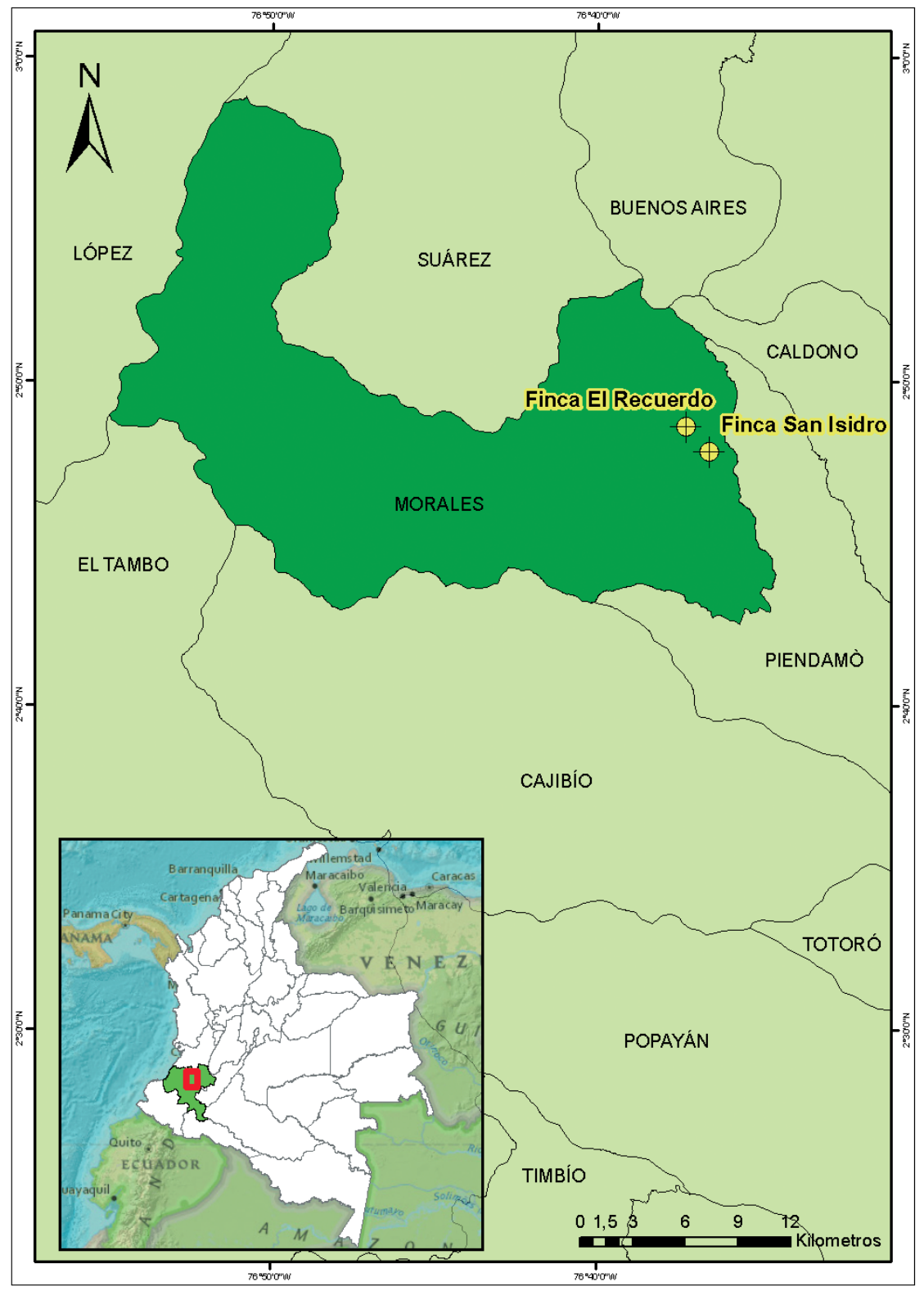

Figura 1. Localización de los huertos El Recuerdo y San Isidro en el municipio de Morales, Cauca, Colombia. 
Visitantes florales y actividad sobre Persea americana cv. Hass. Durante la floración (agosto y septiembre de 2013 y febrero y marzo de 2014), en cada parcela se seleccionaron aleatoriamente 4 árboles. Las observaciones en cada parcela se realizaron 2 veces por semana y en cada día de captura, con intervalos de 20 minutos se recolectaron los insectos que visitaban las flores en cada árbol. Las capturas de los visitantes florales se efectuaron con red entomológica o aspirador bucal, y las muestras, una vez rotuladas, se almacenaron en viales de vidrio para su posterior identificación. Se tuvo un esfuerzo de recolecta de 10 h/día. Al día siguiente, en cada árbol, se registró el comportamiento de los insectos en 10 inflorescencias/árbol durante $2 \mathrm{~min} 30 \mathrm{~s}$ en cada una, entre las 07:00-17:00 h. Se realizó un muestreo rotativo en el que los árboles muestreados se cambiaron cada semana. Se determinó mediante observación directa y registro fotográfico si el insecto hacía contacto con las estructuras reproductivas, el tiempo de permanencia en la flor, el número de flores visitadas (respecto al número de flores abiertas), el recurso floral colectado y la fase floral de acuerdo a la caracterización determinada por Cabezas et al. (2003). Con base a esta información se identificaron las partes del cuerpo del insecto (i.e., cabeza, tórax, abdomen y patas) y se relacionaron con las estructuras reproductivas de las flores, con las cuales entraban en contacto (Figura 2).

Identificación de especies de Diptera. En el análisis de carga de polen, los insectos se individualizaron en viales, se recolectaron visitantes florales de los diferentes órdenes: Coleoptera, Diptera, Hemiptera, Hymenoptera y Lepidoptera. Se realizó el montaje y la curaduría del material en el laboratorio de entomología de Corpoica (actualmente Agrosavia), Centro de Investigación Palmira. La recolecta estuvo amparada por el permiso marco de recolección de especímenes de especies silvestres de la diversidad biológica con fines de investigación científica no comercial, presentado por la Corporación Colombiana de Investigación Agropecuaria (Corpoica) Resolución $\mathrm{N}^{\circ} 1466$ de 03 de diciembre de 2014. La mayoría de los insectos fueron identificados mediante claves taxonómicas de González y Carrejo (1992) y realizando comparaciones con insectos registrados en la colección del Museo de Entomología de la Universidad del Valle (MUSENUV). En el caso de Syrphidae y Calliphoridae se contó con la colaboración de Marta Wolff y Augusto Montoya (Universidad de Antioquia), especialistas en estas familias. En la identificación de Tachinidae se utilizó la clave de Brown et al. (2010). Los insectos colectados, reposan en la Colección Entomológica de Agrosavia Centro de Investigación Palmira.

Carga polínica. Para la determinación de los tipos polínicos, inicialmente se examinó la carga de polen en cada región del cuerpo de los insectos. El polen se retiró con la ayuda de una jeringa de insulina que contenía gelatina glicerinada teñida con fucsina. En la identificación taxonómica de los granos de polen se usó un microscopio óptico con contraste de fases y reglilla ocular micrométrica (Nikon Eclipse E200), catálogos polínicos y atlas palinológicos de Roubik y Moreno (1991) y Giraldo et al. (2011).

Los análisis estadísticos fueron llevados a cabo usando el programa Statistica versión 10. Se evaluó la riqueza, dominancia, equitabilidad y diversidad mediante los índices de Margalef (DMg), Shannon-Weiner $(\mathrm{H})$ e Inverso de Simpson (1-D) encontrados en el programa Past versión 2.17c (Hammer et al., 2001). La eficiencia del muestreo se obtuvo mediante el programa EstimateS versión 9.1.0 (Colwell, 2013). A partir de estimadores no paramétricos se realizaron curvas de acumulación de especies.

\section{Resultados y discusión}

Visitantes florales asociados a Persea americana cv. Hass. Los resultados del estudio revelaron que durante los meses correspondientes a la floración principal (febrero-marzo de 2014) y la denominada "traviesa" (agosto-septiembre de 2013), las flores de aguacate cv. Hass son visitadas en mayor número por insectos pertenecientes a los órdenes 

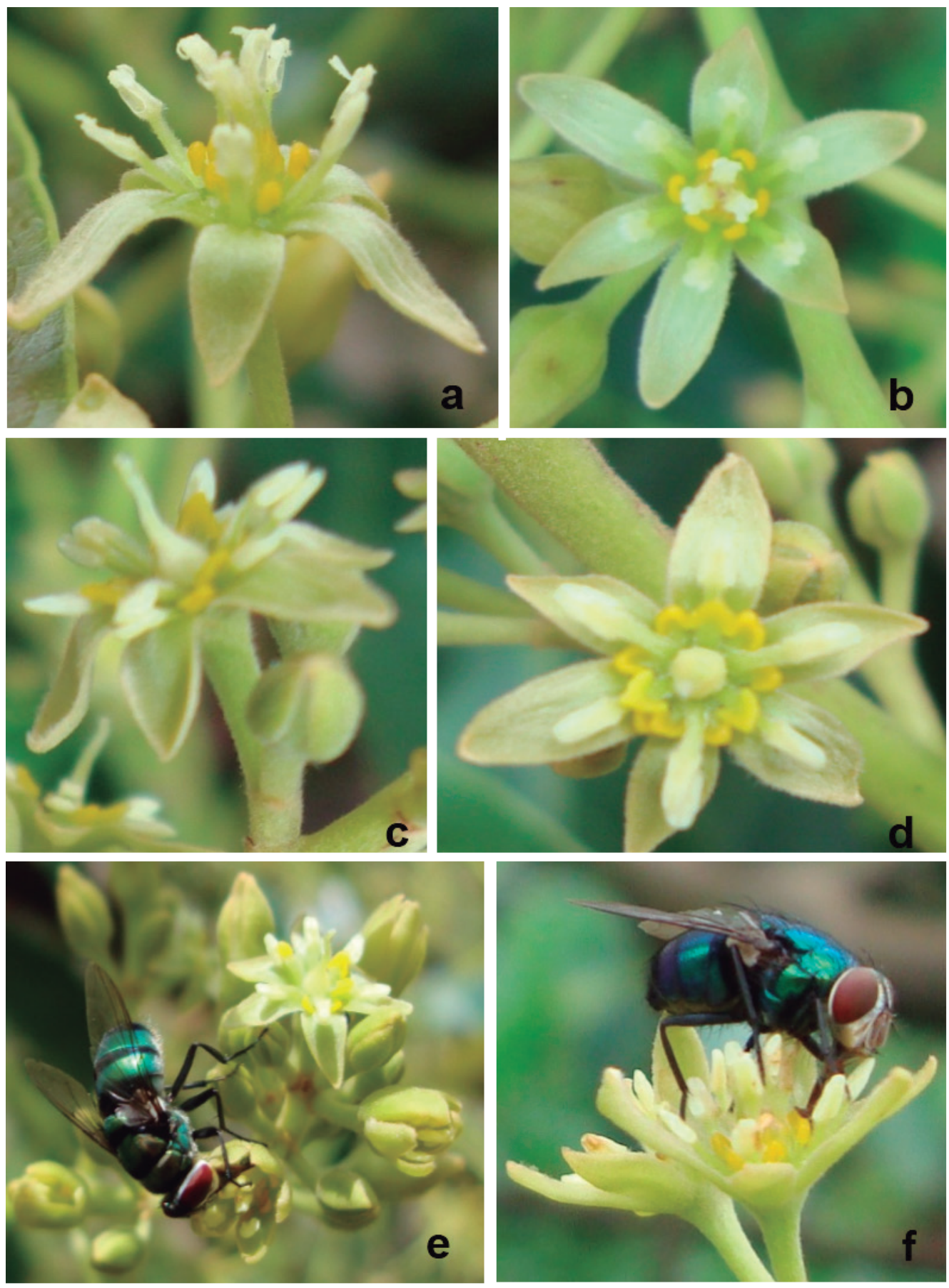

Figura 2. Observación de insectos visitantes y anatomía floral de P. americana cv. Hass. A y B) Flores en fase masculina, C y D) flores en fase femenina. E) Llegada de Chrysomya putoria a la flor, F) llegada de insecto Lucilia eximia. 
Coleoptera, Diptera, Hemiptera, Hymenoptera y Lepidoptera (Tabla 1). Se registró un total de capturas de 974 especímenes, de los cuales la mayoría (82 \%) se colectó en P-1, reunidos en 31 familias. En P-2, se capturó el 18 \% restante (177 insectos), agrupados en 23 familias. El 68,54\% de los Hymenoptera recolectados pertenece a la familia Apidae, seguida de Vespidae (14,51 \%), y Formicidae (7\%); siendo la abeja Apis mellifera el himenóptero más representativo de acuerdo con su abundancia (36,26\%). Respecto a Diptera se destacan las familias Syrphidae (31,6\%), Calliphoridae (33,6 \%), Tachinidae (17,2 \%) y Muscidae (11,2\%). Las especies másrepresentativas de Coleoptera fueron Macrodactylus subspinosus Fabricius (Scarabaeidae) $(4,87 \%)$ y una especie no identificada de Cantharidae $(4,87 \%)$. Los insectos de los órdenes Hemiptera y Lepidoptera tuvieron una baja frecuencia de aparición.

Especies de Diptera asociadas $P$. americana $\mathrm{cv}$. Hass. En este estudio se amplía la información concerniente a Diptera (Tabla 2, Figura 3). Los resultados mostraron que las flores de $P$. americana cv. Hass, son visitadas con mayor frecuencia por especies de la familia Calliphoridae: Lucilia eximia (Wiedemann) (21,6 \%), Chrysomya putoria (Wiedemann) (10,4 \%), y Sirphidae: Palpada scutellaris (Fabricius) (8,8 \%), Allograpta obliqua (Say) (5,6 \%), Ornidia obesa (Fabricius) (7,2 \%), Ornidia major Curran (0,4\%), Palpada vinetorum
(Fabricius) (0,8 \%), Palpada furcata (Wiedemann) $(0,8 \%)$, Ocyptamus lineatus (Macquart) (0,4 \%), Toxomerus watsoni (Curran) (0,8 \%), Copestylum aff. infractum Thompson (1,2\%), Copestylum vagum (Wiedemann) (1,2\%), Toxomerus virgulatus Macquart $(0,8 \%)$ y Toxomerus dispar (Fabricius) $(0,8 \%)$.

Los dípteros visitantes encontrados se caracterizan por presentar adaptaciones anatómicas y comportamentales que influyen en la polinización (Rader et al., 2013; Woodcock et al., 2014). Aunque Bibionidae, Calliphoridae, Muscidae, Syrphidae y Tachinidae han sido reportadas como visitantes florales de diversas plantas, estas familias presentan especímenes con hábitos y preferencias alimenticias diversos (Woodcock et al., 2014). Los Calliphoridae fueron los dípteros más frecuentes, aunque más reconocidos como vectores de enfermedades y causantes de miasis, son importantes polinizadores. En el mango Mangifera indica L., Lucilia spp. y Calliphora spp. (Calliphoridae) incrementan el rendimiento y la calidad del cultivo (Saeed et al., 2016). Se encontró un complejo de la especie L. eximia. Esta mosca tiene distribución neotropical y neártica y ha colonizado principalmente áreas urbanas donde consume frutas, carroña o desechos (Wolff et al., 2010). En el presente estudio se registra por primera vez a esta especie como vector de polen de aguacate Hass en Colombia. También ha sido reportada como polinizadora de Jaborosa rotacea

Tabla 1. Insectos capturados visitando flores de aguacate cv. Hass.

\begin{tabular}{ccccc}
\hline \multirow{2}{*}{ Orden } & \multicolumn{2}{c}{ Parcela 1 } & \multicolumn{2}{c}{ Parcela 2 } \\
\cline { 2 - 5 } & $\begin{array}{c}\text { Número } \\
\text { de familias }\end{array}$ & $\begin{array}{c}\text { Número } \\
\text { de insectos }\end{array}$ & $\begin{array}{c}\text { Número de } \\
\text { familias }\end{array}$ & $\begin{array}{c}\text { Número de } \\
\text { insectos }\end{array}$ \\
\hline Coleoptera & 7 & 96 & 6 & 49 \\
\hline Diptera & 9 & 349 & 8 & 86 \\
\hline Hemiptera & 5 & 10 & 1 & 9 \\
\hline Hymenoptera & 12 & 339 & 5 & 33 \\
\hline Lepidoptera & 2 & 3 & 0 & 0 \\
\hline Total & 35 & 797 & 20 & 177 \\
\hline
\end{tabular}


(Lillo) Hunz y Barboza (Solanaceae) en Argentina (Moré et al., 2013) y Stapelia L. (Apocynaceae) en Brasil (Capote et al., 2014).

Los Sarcophagidae son considerados como moscas necrófagas; los Tachinidae son parasitoides de otros insectos durante su etapa larval; por otra parte, Muscidae tiene hábitos alimenticios omnívoros (Larson et al., 2001; Woodcock et al., 2014). Musca domestica fue un visitante ocasional dado el bajo número de especímenes colectados, en Suráfrica visita las flores del aguacate para el consumo de néctar, careciendo de importancia en la polinización (Eardley y Mansell, 1996).

Los Syrphidae son los únicos reconocidos por libar el néctar floral y ser polinizadores de varias familias botánicas, presentando un patrón de vuelo único, rápido y sensibilidad a la presencia antropogénica (Zamora-Carrillo et al., 2011), por lo que en este estudio posiblemente fueron submuestreados. La pilosidad corporal y la morfología del aparato bucal también influyen en la efectividad de estos dípteros para el transporte de polen. Syrphidae agrupa dos subfamilias con diferentes efectividades como polinizadores, sien- do los miembros de Syrphinae menos efectivos respecto a los Eristalinae, debido a que los primeros presentan un aparato bucal corto que limita el acceso a los estambres no expuestos, baja pilosidad y acicalamiento y consumo de polen frecuente (Zamora-Carrillo et al., 2011). De acuerdo a lo observado en campo el mayor tamaño corporal de algunos Eristalinae (p. ej., Ornidia spp.) determinaba que tuvieran que posarse en la flor para el consumo de polen o néctar, en cambio los Syrphinae (p. ej., Allograpta spp.) en algunas visitas ingresaban lateralmente en la flor consumiendo néctar sin hacer contacto con las anteras (Larson et al., 2001). Este comportamiento ha sido registrado en cultivos de aguacate, lo cual resta importancia como vectores de polen (Eardley y Mansell, 1996; Ish-Am et al. 1999). La morfología floral con las estructuras reproductivas y nectarios expuestos permite que los Syrphidae con probosis de diferente longitud accedan a los recursos florales (Larson et al., 2001). Por otra parte, la constancia de los visitantes florales incrementa su efectividad como polinizadores; sírfidos como Syrphus ribesii (L.) muestran constancia en sus visitas (Woodcock et al., 2014).

Tabla 2. Dípteros asociados a la floración de $P$. americana $\mathrm{cv}$. Hass. V= visitante; $\mathrm{P}=$ polinizador. $\mathrm{N}=$ néctar; $\mathrm{A}=$ apareamiento, $\mathrm{Po}=$ polen.

\begin{tabular}{|c|c|c|c|c|}
\hline Familia & Especie & $\begin{array}{l}\text { Número de } \\
\text { insectos }\end{array}$ & Categoría & $\begin{array}{c}\text { Recurso } \\
\text { colectado }\end{array}$ \\
\hline Asilidae & Ommatius sp. (Wiedemann, 1821) & 1 & $\mathrm{~V}$ & N, Presas \\
\hline \multirow{2}{*}{ Bibionidae } & Dilophus sp. 1 & 2 & $\mathrm{~V}$ & $\mathrm{~N}$ \\
\hline & Dilophus sp. 2 & 1 & V & $\mathrm{N}$ \\
\hline \multirow{3}{*}{ Calliphoridae } & Chrysomya putoria (Wiedemann, 1830) & 26 & $\mathrm{P}$ & $\mathrm{N}$ \\
\hline & Hemilucilia segmentaria (Fabricius, 1805) & 4 & V & $\mathrm{N}$ \\
\hline & Lucilia eximia (Wiedemann, 1819) & 54 & $\mathrm{P}$ & $\mathrm{N}$ \\
\hline \multirow{2}{*}{ Muscidae } & Musca domestica (L., 1758) & 5 & V & $\mathrm{N}$ \\
\hline & Muscidae sp. 2 & 23 & $\mathrm{~V}$ & $\mathrm{~N}$ \\
\hline Sarcophagidae & Peckia anguilla (Curran y Walley, 1934) & 7 & $\mathrm{~V}$ & $\mathrm{~N}$ \\
\hline Syrphidae & Allograpta obliqua (Say, 1823) & 14 & $\mathrm{P}$ & $\mathrm{N}, \mathrm{Po}$ \\
\hline
\end{tabular}


Cont. Tabla 2. Dípteros asociados a la floración de P. americana cv. Hass. V= visitante; P= polinizador. $\mathrm{N}=$ néctar; $\mathrm{A}=$ apareamiento, $\mathrm{Po}=$ polen.

\begin{tabular}{|c|c|c|c|c|}
\hline Familia & Especie & $\begin{array}{l}\text { Número de } \\
\text { insectos }\end{array}$ & Categoría & $\begin{array}{l}\text { Recurso } \\
\text { colectado }\end{array}$ \\
\hline \multirow{16}{*}{ Syrphidae } & Argentinomyia peruvianus (Shannon, 1927) & 2 & $\mathrm{~V}$ & N, Po \\
\hline & Copestylum aff. infractum (Thompson, 1981) & 3 & $\mathrm{~V}$ & N, Po \\
\hline & Copestylum posticum (Say, 1829) & 1 & $\mathrm{~V}$ & N, Po \\
\hline & Copestylum vagum (Wiedemann, 1830) & 3 & $\mathrm{~V}$ & N, Po \\
\hline & Hybobathus lividus (Schiner, 1868) & 1 & $\mathrm{~V}$ & N, Po \\
\hline & Hybobathus persimilis (Curran, 1930) & 2 & $\mathrm{~V}$ & N, Po \\
\hline & Ocyptamus lineatus (Macquart, 1846) & 1 & $\mathrm{~V}$ & N, Po \\
\hline & Ornidia major (Curran, 1930) & 1 & $\mathrm{~V}$ & N, Po \\
\hline & Ornidia obesa (Fabricius, 1775) & 18 & $\mathrm{~V}$ & N, Po \\
\hline & Palpada furcata (Wiedemann, 1819) & 2 & $\mathrm{~V}$ & N, Po \\
\hline & Palpada scutellaris (Fabricius, 1805) & 22 & $\mathrm{P}$ & N, Po \\
\hline & Palpada vinetorum (Fabricius, 1798) & 2 & $\mathrm{~V}$ & N, Po \\
\hline & Salpingogaster nigra (Schiner, 1868) & 1 & $\mathrm{~V}$ & N, Po \\
\hline & Toxomerus dispar (Fabricius, 1794) & 2 & $\mathrm{~V}$ & N, Po \\
\hline & Toxomerus virgulatus (Macquart, 1850) & 2 & $\mathrm{~V}$ & N, Po \\
\hline & Toxomerus watsoni (Curran, 1930) & 2 & $\mathrm{~V}$ & N, Po \\
\hline \multirow{13}{*}{ Tachinidae } & Archytas sp. (Jaennicke, 1867) & 2 & $\mathrm{~V}$ & $\mathrm{~N}$ \\
\hline & Eujuriniodes sp. 1 (Townsend, 1935) & 4 & $\mathrm{~V}$ & $\mathrm{~N}$ \\
\hline & Gonia sp. (Meigen, 1803) & 1 & $\mathrm{~V}$ & $\mathrm{~N}$ \\
\hline & Hystricia sp. (Macquart, 1844) & 1 & $\mathrm{~V}$ & $\mathrm{~N}$ \\
\hline & Leschenaultia sp. 1 (Robineau-Desvoidy, 1830) & 17 & $\mathrm{~V}$ & $\mathrm{~N}$ \\
\hline & Leschenaultia sp. 2 (Robineau-Desvoidy, 1830) & 11 & $\mathrm{~V}$ & $\mathrm{~N}$ \\
\hline & Parepalpus sp. 1 (Coquillett, 1902) & 1 & $\mathrm{~V}$ & $\mathrm{~N}$ \\
\hline & Quadratosoma sp. 1 (Townsend, 1914) & 1 & $\mathrm{~V}$ & $\mathrm{~N}$ \\
\hline & Quadratosoma sp. 2 (Townsend, 1914) & 1 & $\mathrm{~V}$ & $\mathrm{~N}$ \\
\hline & Rhachoepalpus sp. (Townsend, 1908) & 1 & $\mathrm{~V}$ & $\mathrm{~N}$ \\
\hline & Trichophora sp. (Macquart, 1847) & 1 & V & $\mathrm{N}$ \\
\hline & Trichopoda sp. (Berthold, 1827) & 1 & $\mathrm{~V}$ & $\mathrm{~N}$ \\
\hline & Xanthoepalpus sp. (Townsend, 1914) & 1 & $\mathrm{~V}$ & $\mathrm{~N}$ \\
\hline \multirow{3}{*}{ Tephritidae } & Anastrepha fraterculus (Wiedemann, 1830). & 1 & $\mathrm{~V}$ & $\mathrm{~N}$ \\
\hline & Anastrepha striata (Schiner, 1868) & 3 & $\mathrm{~V}$ & $\mathrm{~N}$ \\
\hline & Euaresta sp. (Hendel, 1914) & 1 & $\mathrm{~V}$ & $\mathrm{~N}$ \\
\hline
\end{tabular}



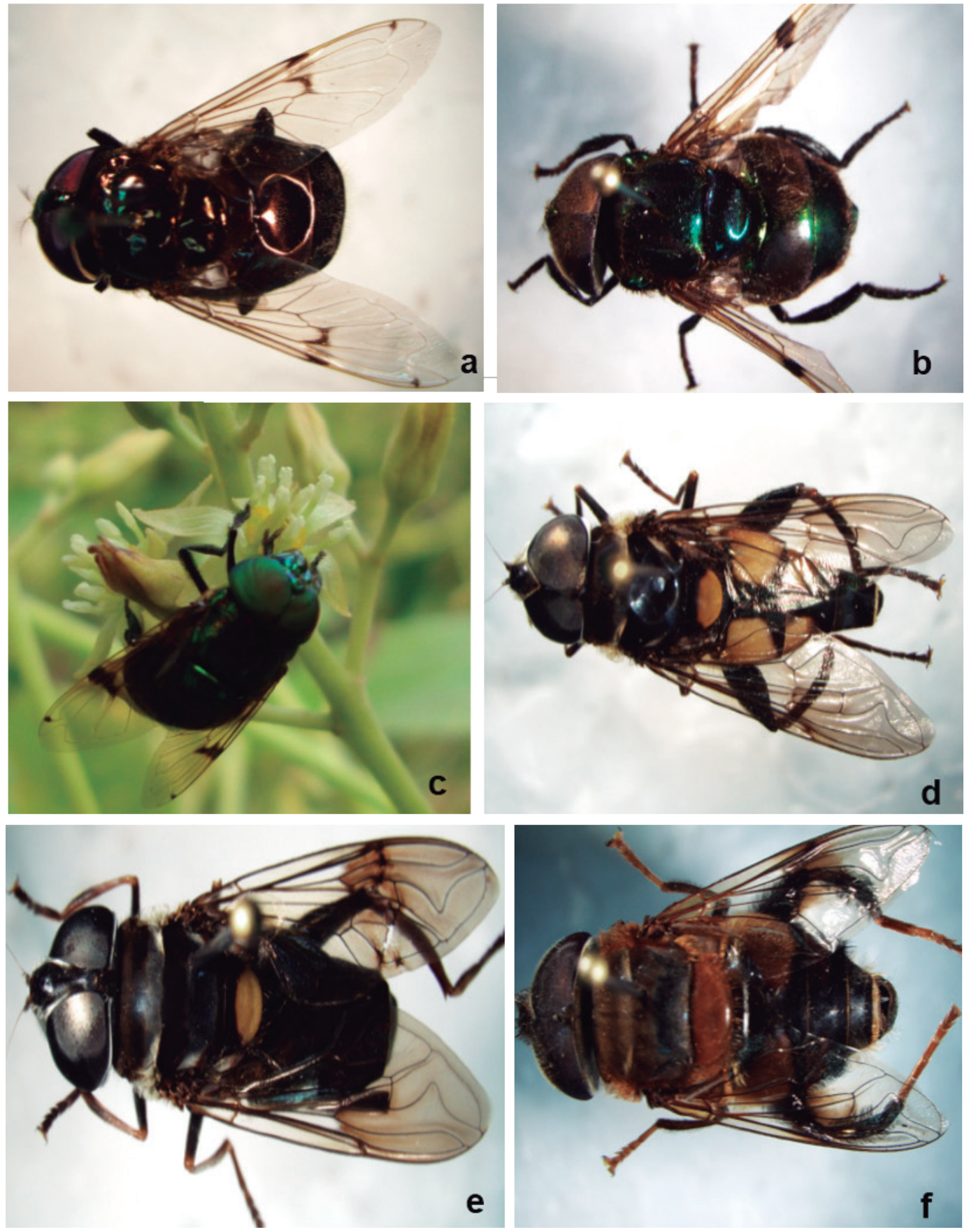

Figura 3. Dípteros asociados a P. americana cv. Hass. A y B) Ornidia obesa. C) O. major. D y E) Palpada scutellaris. F) P. vinetorum. G) Palpada furcate. H) Ocyptamus lineatus. I) Copestylum aff. Infractum. J) C. vagum. K) Toxomerus watsoni. L) Leschenaultia sp. 1 M) Toxomerus dispar. N) T. virgulatus. O) Allograpta obliqua. P) Lucilia eximia. Q) Chrysomya putoria. R) Musca domestica. 

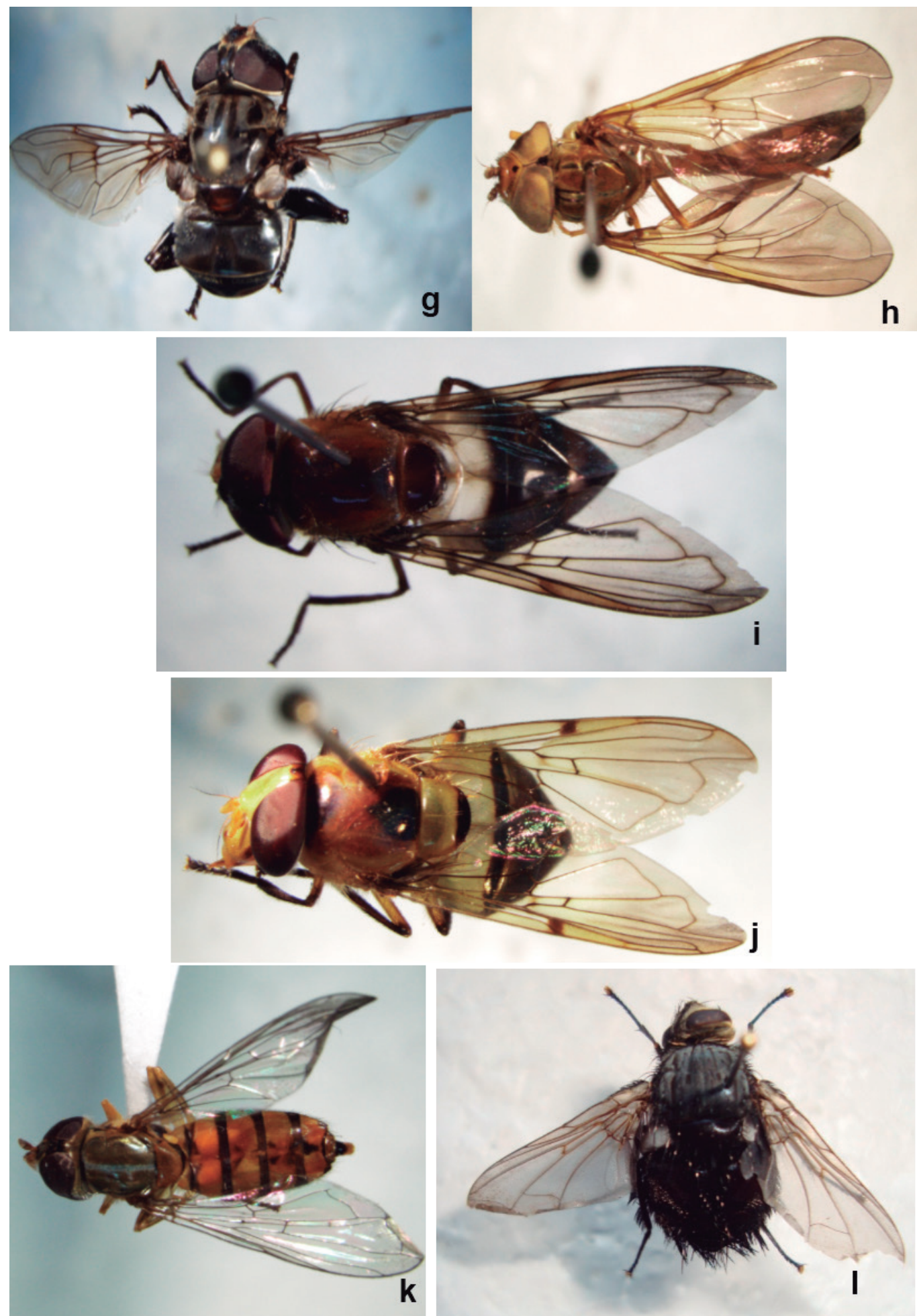

Figura 3. (Cont.) Dípteros asociados a P. americana cv. Hass. A y B) Ornidia obesa. C) O. major. D y E) Palpada scutellaris. F) P. vinetorum. G) Palpada furcate. H) Ocyptamus lineatus. I) Copestylum aff. Infractum. J) C. vagum. K) Toxomerus watsoni. L) Leschenaultia sp. 1. M) Toxomerus dispar. N) T. virgulatus. O) Allograpta obliqua. P) Lucilia eximia. Q) Chrysomya putoria. R) Musca domestica. 

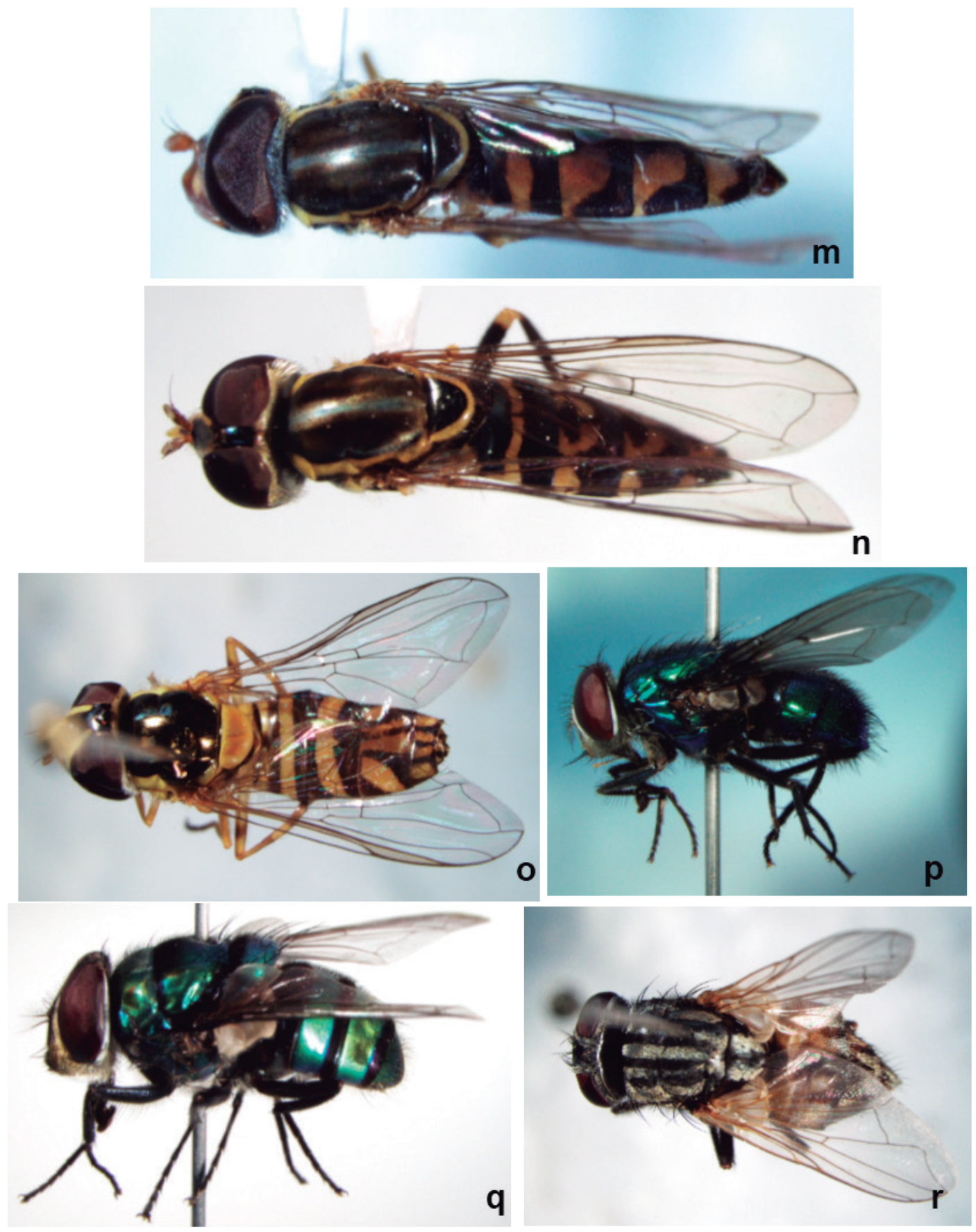

Figura 3. (Cont.) Dípteros asociados a P. americana cv. Hass. A y B) Ornidia obesa. C) O. major. D y E) Palpada scutellaris. F) P. vinetorum. G) Palpada furcate. H) Ocyptamus lineatus. I) Copestylum aff. Infractum. J) C. vagum. K) Toxomerus watsoni. L) Leschenaultia sp. 1. M) Toxomerus dispar. N) T. virgulatus. O) Allograpta obliqua. P) Lucilia eximia. Q) Chrysomya putoria. R) Musca domestica. 
Los resultados de esta investigación coinciden con lo reportado por Castañeda-Vildózola et al. (1999) en México en cuanto al número de especies de díptera presentes en las parcelas, y también se presenta correspondencia con los géneros reportados en este estudio. Mencionan de la familia Syrphidae los géneros: Allograpta, Copestilum, Hemilucila, Chrysomya, Ornidia, Palpada y Toxomerus como visitantes florales del cultivo. También de Tachinidae: Archytas, Hystricia y Leschenaultia. Siendo el Calliphoridae Chrysomya megachepala un potencial polinizador secundario, que transporta la mayor cantidad de polen en la región ventral del cuerpo y las patas. Los dípteros son el segundo grupo de importancia para la polinización del cultivo después de Hymenoptera, debido a su diversidad y abundancia (CastañedaVildózola et al., 1999). Sin embargo, varias especies presentan cantidades mínimas de polen o una baja tasa de visita floral, por lo cual estos son considerados visitantes florales sin aporte en la polinización (Eardley y Mansell, 1996; CastañedaVildózola et al., 1999; Ish-Am et al., 1999).

Actividad de visitantes florales. El registro de la actividad de los insectos en las flores está relacionado con los periodos de apertura de las flores en fase masculina y femenina. Las flores de $P$. americana cv. Hass, inician la apertura floral entre las 07:00-08:00 horas en fase femenina $y$ dependiendo de las condiciones climáticas que influyen en los flujos fenológicos, permanece en esta fase, hasta su cierre a las 12:30 horas. En fase masculina las flores abren entre 11:00, a las 13:00, transcurrido este periodo todas las flores abiertas se encuentran en esta fase y hacia las 17:00 la mayoría de ellas están cerradas. El traslape entre fases florales tiene una duración de 2 horas (Figura 4).

Los resultados de los registros de captura mostraron que entre las 07:00-09:00 horas se colectó el 18,82 \% de los especímenes dípteros, coincidiendo con la fase de antesis floral. Durante la apertura floral en fase femenina, (10:00 y 12:00) se obtuvo el 39,5\%. Sin embargo, un incremento $(41,7 \%)$ se registró entre las 13:00 y 16:00 horas (Figura 4). En síntesis, los resultados sugieren que los insectos del orden Díptera presentan una mayor preferencia (58 \%) por visitar las flores de $P$. americana en horas de la mañana (07:00-12:00), comparada con las realizadas en la tarde $(41,7 \%)$.

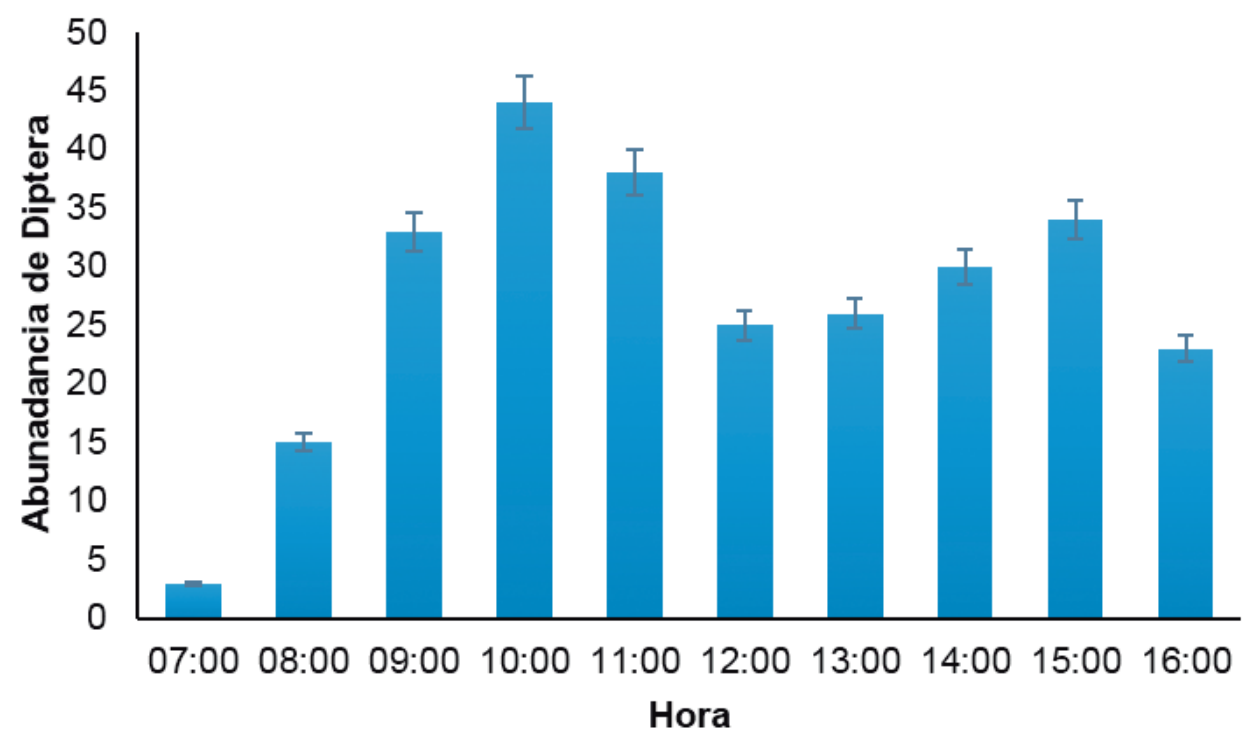

Figura 4. Frecuencia de visita de dípteros asociados a las flores de P. americana cv. Hass, a través del día. 
El seguimiento al comportamiento de los visitantes en las flores de P. americana reveló que, en general, una vez los insectos llegan a las flores, sus actividades estuvieron concentradas en: consumo de néctar, consumo de polen, utilización como plataforma sexual y cópula o forrajeo conjugado con permanencia en la flor durante cortos periodos de tiempo. Las especies de dípteros visitaron un promedio de dos flores por inflorescencia. Sin embargo, alta variabilidad en la duración del tiempo de visita a las flores se encontraron en insectos de la familia Bibionidae, quienes permanecieron mayor tiempo en la flor (59 s), principalmente en cópula o consumiendo néctar (Tabla 3). El grupo de los Muscidae presentó el rango más amplio en el tiempo de permanencia en la flor (2- $40 \mathrm{~s})$, generalmente se encontraron consumiendo néctar o posados en las flores. Los Tachinidae, permanecen en promedio $12 \mathrm{~s}$ y debido a su mayor tamaño, durante la visita tocan las estructuras reproductivas con el área ventral del tórax y las patas (Tabla 3) (Figura 5).

Los Syrphidae en el 68,2 \% de las visitas permanecieron consumiendo néctar, sin hacer contacto con las estructuras reproductivas. Este comportamiento es favorecido por su capacidad de permanecer en vuelo en un mismo punto y la disposición floral con los nectarios expuestos (Tabla 3). Los insectos de los géneros Copestylum, Allograpta y Toxomerus ingresan lateralmente a la flor y permanecen posados sobre la flor entre 3 a 60 s. Sin embargo, pueden consumir el néctar suspendidos en vuelo (Figura 5). En flores masculinas, las anteras entran en contacto con la parte frontal de la cabeza. Los Ornidia spp. y Palpada spp. generalmente se posan sobre la flor y su cabeza y patas hacen contacto con los estambres y el pistilo (Figura 5).

Los dípteros Sarcophagidae y Calliphoridae permanecieron el menor tiempo (entre 8-9 s) en cada flor (Tabla 3). Los Calliphoridae fueron observados consumiendo néctar, tuvieron alta actividad durante la floración intermedia ("travesía") y cuando la actividad de A. mellifera era baja. Así mismo, se observó que los visitantes Calliphoridae exhiben una mayor actividad en días con bajo brillo solar y en los días lluviosos se esconden en el envés de las hojas.

Durante las observaciones los insectos no mostraron preferencia por una fase floral específica; ellos visitaron en proporciones similares inflorescencias con flores en fase femenina y masculina. Las flores de P. americana tienen una disponibilidad permanente de néctar, durante la fase floral femenina este recurso es secretado por los estaminodios, mientras que en la fase masculina los nectarios cumplen esta función (Davenport, 1986; Wysoki et al., 2002). Esta característica podría ser una adaptación a la dicogamia para garantizar la visita de insectos durante las dos fases florales $y$, en consecuencia, alcanzar una efectiva polinización.

Los resultados de este estudio sugieren que las especies presentan una mayor preferencia por visitar flores abiertas, es decir, con estructuras reproductivas expuestas, comportamiento, igualmente descrito por Woodcock et al. (2014), quienes subrayan que los dípteros tienen habilidades limitadas para manipular las flores durante sus visitas. Además, las flores de $P$. americana presentan una morfología y coloración atrayente para Diptera ya que son pequeñas, circulares, abiertas y tienen pétalos y sépalos de coloración amarillo-verdosa y estructuras secretoras de néctar de color amarillo (Davenport, 1986; Peña, 2003). Se ha documentado que dípteros como Eristalis tenax muestran preferencia por flores amarillas (Woodcock et al., 2014). Por otra parte, los dípteros pueden tener una actividad diferencial de acuerdo con la fase floral en plantas protóginas. El díptero Elachiptera formosa Loew (Chloropidae) se aparea en inflorescencias en fase femenina y realiza consumo de polen en flores en fase masculina (Woodcock et al., 2014). 

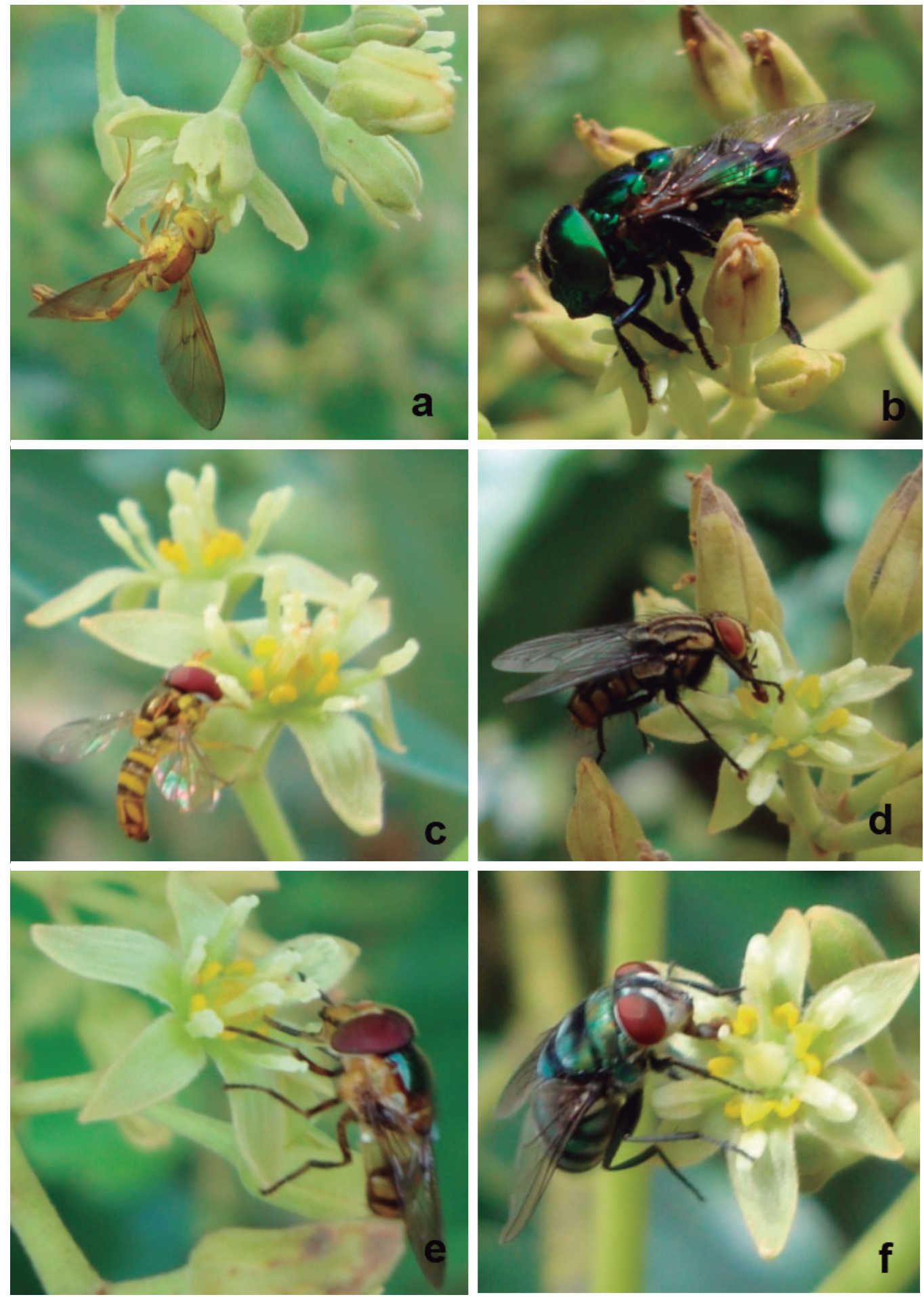

Figura 5. Comportamiento de forrajeo de moscas en flores de P. americana. A) Ocyptamus lineatus. B) Ornidia obesa. C) Allograpta obliqua. D) Peckia anguilla. E) Copestylum aff. infractum. F) Chrysomya putoria. 
Tabla 3. Comportamiento de forrajeo de los dípteros visitantes florales de P. americana cv. Hass en huertos de Morales, Cauca.

\begin{tabular}{|c|c|c|c|c|c|c|c|}
\hline \multirow[t]{2}{*}{ ORDEN/FAMILIA } & \multirow{2}{*}{$\begin{array}{c}\text { Número } \\
\text { de insectos } \\
\text { observados }\end{array}$} & \multicolumn{2}{|c|}{$\begin{array}{l}\text { Tiempo de visita } \\
\text { por flor }\end{array}$} & \multicolumn{2}{|c|}{$\begin{array}{c}\text { Flores } \\
\text { visitadas por } \\
\text { inflorescencia }\end{array}$} & \multirow{2}{*}{$\begin{array}{c}\text { Contacto con } \\
\text { estructuras } \\
\text { masculinas } \\
(\%)\end{array}$} & \multirow{2}{*}{$\begin{array}{c}\text { Contacto con } \\
\text { estructuras } \\
\text { femeninas } \\
(\%)\end{array}$} \\
\hline & & Promedio & S.E. & Promedio & S.E. & & \\
\hline \multicolumn{8}{|l|}{ Diptera } \\
\hline Bibionidae & 6 & 59,20 & 23,35 & 2,5 & 0,50 & 66,67 & 66,67 \\
\hline Calliphoridae & 67 & 8,77 & 0,81 & 2,2 & 0,23 & 50,75 & 29,85 \\
\hline Muscidae & 12 & 12,65 & 3,18 & 1,8 & 0,43 & 41,67 & 33,33 \\
\hline Syrphidae & 22 & 10,13 & 2,73 & 1,3 & 0,14 & 31,82 & 31,82 \\
\hline Sarcophagidae & 18 & 8,53 & 1,54 & 1,6 & 0,18 & 33,33 & 27,78 \\
\hline Tachinidae & 12 & 12,10 & 5,58 & 1,8 & 0,46 & 8,33 & 8,33 \\
\hline
\end{tabular}

En todas las especies de dípteros se encontraron granos de polen. Allograpta obliqua presentó un 39 \% más de polen, comparada con Palpada scutellaris, la segunda mejor especie portadora de polen. Estos resultados fueron consistentes para A. obliqua cuando se comparó el número de granos de polen de P. americana (119 granos), especie que junto a Hemilucilia segmentaria y Muscidae sp. 2 transportan el mayor número de granos de polen de aguacate (Tabla 4). Con excepción de Hybobathus lividus, Leschenaultia sp. 2 y Ornidia obesa, las especies de Calliphoridae registradas en este estudio llevan en su cuerpo polen de $P$. americana, sugiriendo, que C. putoria, H. segmentaria y L. eximia y del Muscidae sp. 2, por su mayor carga polínica podrían estar jugando un rol importante en la actividad polinizadora de $P$. americana cv. Hass.

Así mismo, los resultados muestran una alta diversidad de insectos asociada a la floración $P$. americana cv. Hass. Los resultados en este estudio son consistentes, al ser comparados con estudios previos realizados en México (Castañeda-Vildózola et al., 1999; Pérez-Balam et al., 2012), quienes revelan que los visitantes florales pertenecen a varios gremios tróficos y coinciden en la importancia de algunas especies como vectores de polen.

La carga de polen indica que estas especies visitan otras especies vegetales que complementen su dieta, en su mayoría el consumo de recursos alimenticios ricos en carbohidratos, como el néctar, considerado una fuente energética de importancia por su contenido de azúcares, lípidos y aminoácidos para actividades como el vuelo, dispersión, reproducción en especial para desarrollo ovárico y producción de huevos (Rader et al., 2013). También encuentran recursos no nutritivos, ya que las flores ofrecen protección contra las inclemencias climáticas, sitios de apareamiento y atracción para presas de depredadores (Woodcock et al., 2014). Estos recursos vegetales alternativos al cultivo podrían garantizar la permanencia de los visitantes florales en los huertos durante la floración. En Michoacán se atribuye la baja diversidad y abundancia de polinizadores del cultivo a la destrucción de los sitios de refugio y anidación por la deforestación y aplicación de insecticidas de amplio espectro (Castañeda-Vildózola et al., 1999). 
Tabla 4. Carga de polen de dípteros visitantes florales del aguacate cv. Hass

\begin{tabular}{lcccc}
\hline \multicolumn{1}{c}{ Especie } & $\begin{array}{c}\text { Número de } \\
\text { insectos }\end{array}$ & $\begin{array}{c}\text { Número total } \\
\text { de granos de } \\
\text { polen }\end{array}$ & $\begin{array}{c}\text { Número de } \\
\text { granos de polen } \\
\text { de aguacate cv. } \\
\text { Hass }\end{array}$ & $\begin{array}{c}\text { Porcentaje } \\
\text { de polen de } \\
\text { aguacate cv. } \\
\text { Hass }\end{array}$ \\
\hline Allograpta obliqua & 5 & 1868 & 119 & 6,37 \\
\hline Chrysomya putoria & 5 & 362 & 53 & 14,64 \\
\hline Hemilucilia segmentaria & 3 & 135 & 120 & 88,88 \\
\hline Hybobathus lividus & 2 & 445 & 0 & 0 \\
\hline Leschenaultia sp.1 & 2 & 126 & 44 & 34,92 \\
\hline Leschenaultia sp. 2 & 2 & 141 & 0 & 0 \\
\hline Lucilia eximia & 17 & 111 & 27 & 24,32 \\
\hline Muscidae sp. 2 & 11 & 119 & 88 & 73,95 \\
\hline Ornidia obesa & 2 & 169 & 0 & 0 \\
\hline Palpada scutellaris & 4 & 729 & 6 & 0,82 \\
\hline
\end{tabular}

Los visitantes florales presentaron una alta diversidad y baja frecuencia de aparición de las especies. Se presentaron significativas diferencias en la diversidad, de acuerdo con el índice de Shannon y la riqueza de especies P-1 fue mayor (Tabla 5).

Tabla 5. Análisis de la diversidad de dípteros entre las parcelas estudiadas.

\begin{tabular}{lcc}
\hline \multicolumn{1}{c}{$\begin{array}{c}\text { Índices de diversidad y } \\
\text { abundancia }\end{array}$} & Parcela 1 & Parcela 2 \\
\hline Margalef (DMg) & 7,77 & 3,90 \\
\hline Shannon-Weiner (H) & 2,92 & 2,49 \\
\hline Inverso de Simpson (1-D) & 0,9095 & 0,9056 \\
\hline $\begin{array}{l}\text { Número de especies o } \\
\text { riqueza específica (S) }\end{array}$ & 44 & 14 \\
\hline
\end{tabular}

Se registraron 44 especies de Diptera en P-1; sin embargo, los estimadores de riqueza las curvas CHAO 1 y ACE sugieren la existencia de 65-71 especies, respectivamente. Los estimadores de riqueza no llegaron a la asíntota, lo que sugiere que el número de especies presentes debe ser mayor al encontrado (Figura 6). Se obtuvo una eficiencia de muestreo del $67,7 \%$.

Se registraron 14 especies de Díptera en P-2. Los estimadores de riqueza $\mathrm{CHAO} 1$ y $\mathrm{ACE}$ indican que deben encontrarse entre 17-19 especies en la zona (Figura 7). Se obtuvo una eficiencia del muestreo del 82,35\%.

Los análisis indican que el muestreo fue más eficiente en P-2, lo que podría deberse a que hubo menor ocurrencia de especies raras. Debido a que el número de especies registradas depende del tamaño de la muestra, al incrementar los muestreos es más probable encontrar especies raras (Moreno, 2001). Así, en P-1 el registro de un mayor número de especies con una baja frecuencia de aparición determinó que las curvas de los estimadores no alcanzaran una asíntota, dado que estos indicadores son sensibles a las especies raras.

El hecho que las curvas de diversidad no alcancen una asíntota es un indicativo de la alta 
diversidad de insectos que se puede encontrar en estos agroecosistemas durante el periodo de floración. Si bien la diversidad en términos de la relación entre riqueza y abundancia solo permite comparar las áreas de interés durante un periodo específico (Rader et al., 2013), estos estimadores son de fácil obtención e informativos.
Las diferencias encontradas entre parcelas podrían estar determinadas por las condiciones de hábitat en términos de ofertas de recursos florales y de nidificación, ya que las comunidades de polinizadores son sensibles a estas condiciones, incluso pueden variar a lo largo de tiempo y el espacio (Rader et al., 2013; Saeed et al., 2016).

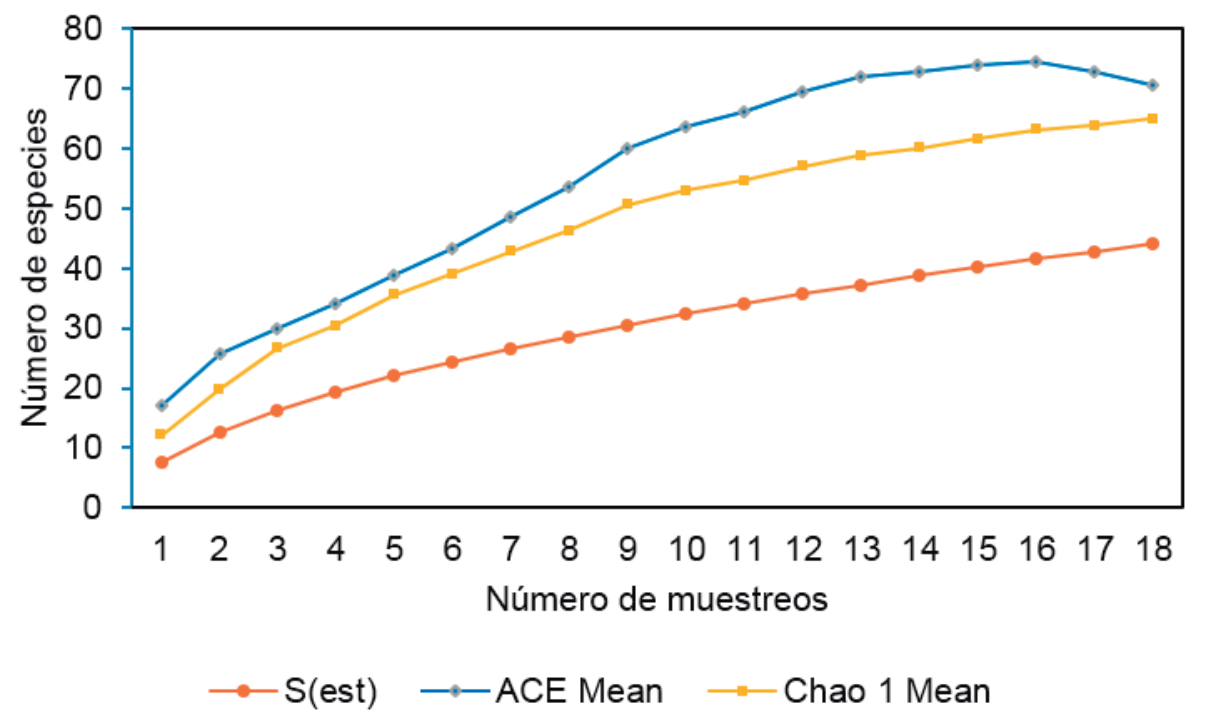

Figura 6. Curva de acumulación de especies de Díptera observadas en P-1.

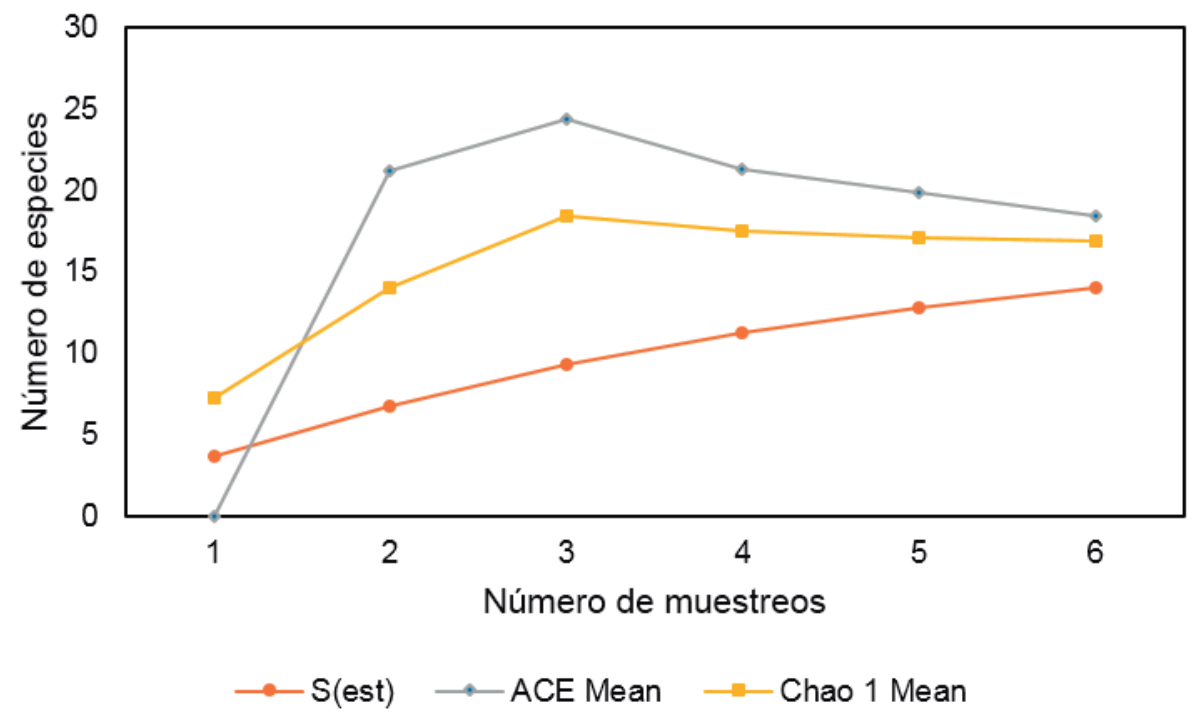

Figura 7. Curva de acumulación de especies de Diptera observadas en P-2. 
En las inflorescencias marcadas entre el 12-38 $\%$ de las flores abiertas (i.e., disponibles para polinización) fueron visitadas. $P$. americana produce cerca de 1000000 de flores (Wysoki et al., 2002), pero el comportamiento de los insectos en la flor y el tiempo de permanencia son factores que podrían estar modulando el número de flores polinizadas.

En síntesis, los resultados de este estudio constituyen el primer reporte de la diversidad de dípteros que son visitantes florales del cultivo $P$. americana, y revelan su desconocido papel como potenciales polinizadores de especies que cumplen otras funciones biológicas. Se resalta la importancia de la conservación de la flora nativa como fuente de refugio y complemento nutricional de una alta diversidad de insectos de distintos gremios tróficos asociados a la floración que contribuyen diferencialmente a la polinización de P. americana var. Hass.

\section{Agradecimientos}

Los autores agradecen a la profesora Martha Wolff y Augusto Montoya (Universidad de Antioquia) por la identificación de las especies de Diptera. A los biólogos Oscar Ascuntar, Diana Torres (Universidad del Valle) por su colaboración en la identificación de Hymenoptera, Coleoptera y Hemíptera como también a Carmen Elisa Posso, curadora del Museo de Entomología de la Universidad del Valle, por facilitar la revisión y comparación de especímes; a la asociación Asproamor, especialmente a los productores Fernando Velasco y Aldemar Sarria por permitir realizar la investigación en sus predios. A Sandra Yaneth Pinchao por su apoyo en los análisis palinológicos. A la Corporación Colombiana de Investigación Agropecuaria (Corpoica, actualmente Agrosavia), C.I Palmira. Esta investigación se desarrolló en el marco del proyec-to: "Manejo Integrado del Cultivo de Aguacate (Persea americana) cv. Hass para Incrementar la Calidad y el Rendimiento en Zonas Productoras de Colombia", financiado por Colciencias. Finalmente, a los evaluadores anónimos quienes, con sus aportes, enriquecieron el manuscrito.

\section{Referencias}

Brown, B. V., Borkent, A., Cumming, J. M., Wood, D. M., Woodley, N. E. y Zumbado, M. A. (Eds.). (2010). Manual of Central American Diptera: Volume 2. Ottawa, Ontario, Canadá: NRC Research Press. 728 p.

Cabezas, C., Hueso, J. J. y Cuevas, J. (2003). Identificación y descripción de los estados fenológicos-tipo del aguacate (Persea americana Mill.). Proceedings V World Avocado Congress (Actas V Congreso Mundial del Aguacate), 237-242.

Capote, T., Arcaya, E. y Velásquez, Y. (2014). First report of Lucilia eximia (Wiedemann, 1819) (Diptera: Calliphoridae) associated with Stapelia gigantea L. (Apocynaceae) in Venezuela. Entomotropica, 29(1), 53-56.

Castañeda-Vildózola, A., Equihua, A., Valdés, J., Barrientos, A., Ish-Am, G. y Gazit, S. (1999). Insectos polinizadores del aguacatero en los estados de México y Michoacán, México. Revista Chapingo. Serie Horticultura, 5 (Número Especial), 129-136.

Colwell, R. K. (2013). EstimateS: Statistical estimation of species richness and shared species from samples. Version 9.1.0. Recuperado de http://viceroy.Eeb.Uconn.edu/estimates Revisado el 1 de febrero de 2016.

Davenport, T. L. (1986). Avocado flowering Vol. 8. En Janick, J. (Ed.) Horticultural Reviews. Pp: 257289. Westport, CN: AVI Publishing Co., Inc.

Eardley, C. D. y Mansell, M. W. (1996). The natural occurrence of insect pollinators in an avocado orchard. South African Avocado Growers' Association Yearbook, 19, 36-38.

Giraldo, C., Rodríguez, A., Chamorro, F., Obregón, D., Montoya, P., Ramírez, N., Solarte, V. y Nates-Parra, G. (2011). Guía Ilustrada de polen y plantas nativas visitadas por abejas. Bogotá D.C.: Universidad Nacional de Colombia. 230 p.

González, R. y Carrejo, N. S. (1992). Introducción al estudio de los Diptera. Cali: Centro Editorial Universidad del Valle. 197 p. 
Hammer, O., Harper, D. A. T. y Ryan, P. D. (2001). PAST: Paleontological statistic software package for education and data analysis. Palaeontologia Electronica, 4(1), 1-9.

Inouye, D. W., Larson, B. M. H., Ssymank, A. y Kevan, P. G. (2015). Flies and flowers III: Ecology of foraging and pollination. Journal of Pollination Ecology, 16(16), 115-133.

Ish-Am, G., Barrientos-Priego, F., CastañedaVildozola, A. y Gazit, S. (1999). Avocado (Persea americana Mill.) pollinators in its region of origin. Revista Chapingo Serie Horticultura, 5, 137-143.

Larson, B. H. M., Kevan, P. G. y Inouye, D. W. (2001). Flies and flowers: taxonomic diversity of anthophiles and pollinators. The Canadian Entomologist, 133, 439-465.

Moré, M., Cocucci, A. A. y Raguso, R. A. (2013). The importance of oligosulfides in the attraction of fly pollinators to the brood-site deceptive species Jaborosa rotacea (Solanaceae). International Journal of Plant Sciences, 174(6), 863-876.

Moreno, C. E. (2001). Métodos para medir la biodiversidad. Zaragoza: M\&T-Manuales y Tesis SEA, vol.1. 84 p.

Peña, J. E. (2003). Insectos polinizadores de frutales tropicales: no solo las abejas llevan la miel al panal. Manejo Integrado de Plagas y Agroecología (Costa Rica), 69, 6-20.

Pérez-Balam, J., Quezada-Euán, J. J. G., AlfaroBates, R., Medina, S., McKendrick, L., Soro, A. y Paxton, R. J. (2012). The contribution of honeybees, flies and wasp to avocado (Persea americana) pollination in southern Mexico. Journal of Pollination Ecology, 8(6), 42-47.
Rader, R., Edwards, W., Westcott, D.A., Cunningham, S.A. y Howlett, B.G. (2013). Diurnal effectiveness of pollination by bees and flies in agricultural Brassica rapa: Implications for ecosystem resilience. Basic and Applied Ecology, 14(1), 20-27.

Roubik, D. W. y Moreno, J. E. (1991). Pollen and Spores of Barro Colorado Island. St. Louis, Missouri: Monographs in Systematic Botany No. 36. Missouri Botanical Garden. 268 pp.

Saeed, S., Naqqash, M. N., Jaleel, W., Saeed, Q., y Ghouri, F. (2016). The effect of blow flies (Diptera: Calliphoridae) on the size and weight of mangos (Mangifera indica L.). PeerJ 4:e2076; DOI 10.7717/ peerj.2076.

Wolff, M. I., Rivera, C., Herrera, S. E., Wolff, J. C. (2010). Lucilia eximia (Diptera: Calliphoridae), una nueva alternativa para la terapia larval y reporte de casos en Colombia. Iatreia, 23(2), 107-116.

Woodcock, T. S., Larson, B. H. M., Kevan, P. G., Inouye, D. W. y Lunau, K. (2014). Flies and flowers II: floral attractants and rewards. Journal of Pollination Ecology, 12(8), 63-94.

Wysoki, M., van den Berg, M. A., Ish-Am, G., Gazit, S., Peña, J. E. y Waite, G. K. (2002). Pests and pollinators of avocado. En Peña, J.E., Sharp, J.L. y Wysoki, M. (Eds.). Tropical Fruit Pests and Pollinators: Biology, Economic Importance, Natural Enemies, and Control. Pp: 265-272. CABI.

Zamora-Carrillo, M., Amat-García, G. D. y Fernández-Alonso, J. L. (2011). Estudio de las visitas de las moscas de las flores (Diptera: Syrphidae) en Salvia bogotensis (Lamiaceae) en el jardín botánico José Celestino Mutis (Bogotá D.C., Colombia). Caldasia, 33(2), 453-470. 


\section{Diana Carabalí-Banguero}

Universidad del Valle

Cali, Colombia

dianajohana16@hotmail.com

\section{James Montoya-Lerma}

Universidad del Valle

Cali, Colombia

james.montoya@correounivalle.edu.co

\section{Arturo Carabalí-Muñoz}

Agrosavia-Palmira

Palmira, Colombia

acarabali@corpoica.org.co
Dípteros asociados a la floración del aguacate Persea americana Mill cv. Hass en Cauca, Colombia

Citación del artículo: Carabalí-Banguero, D., Montoya-Lerma, J. y Carabalí-Muñoz, A. (2018). Dípteros asociados a la floración del aguacate Persea americana Mill cv. Hass en Cauca, Colombia. Biota Colombiana, 19(1), 92-111. DOI: 10.21068/ c2018v19n01a06.

Recibido: 1 de septiembre de 2016

Aprobado: 23 de mayo de 2018 